\title{
Host Galaxies of Obscured Quasars: Infant Starbursts Caught in Action
}

\author{
Xin Liu ${ }^{1}$, Nadia L. Zakamska ${ }^{2,3}$, Jenny E. Greene ${ }^{1,4}$, \\ Michael A. Strauss ${ }^{1}$, Julian H. Krolik ${ }^{5}$, and Timothy M. Heckman ${ }^{5}$ \\ ${ }^{1}$ Department of Astrophysical Sciences, Princeton University, Peyton Hall, Ivy Lane, \\ Princeton, NJ 08544, USA \\ Email: xinliu@astro.princeton.edu \\ ${ }^{2}$ Institute for Advanced Study, Einstein Dr., Princeton, NJ 08540, USA \\ ${ }^{3}$ Spitzer Fellow, John N. Bahcall Fellow \\ ${ }^{4}$ Hubble Fellow, Princeton-Carnegie Fellow \\ ${ }^{5}$ Department of Physics and Astronomy, Johns Hopkins University, Baltimore, MD 21218, USA
}

Keywords. galaxies: active, galaxies: evolution, galaxies: interactions, galaxies: nuclei, galaxies: starburst, galaxies: stellar content, quasars: general

Supermassive black holes (SMBHs) in the centers of massive galaxies are thought to predominantly grow in brief Eddington-rate quasar phases accompanied by starbursts, but on-going starbursts in luminous quasars are difficult to observe. Buried under the natural coronagraph, obscured quasars offer a unique window for direct, robust host-galaxy spectroscopy otherwise virtually inaccessible for luminous quasars. Our pilot study at $z \sim 0.5$ (Liu et al. 2009) revealed a substantial contribution from very young stellar populations with ages less than $\sim 100 \mathrm{Myr}$ in all of the observed host galaxy spectra. More dramatically, in three out of the nine SDSS quasars observed, we have witnessed strong infant starbursts with ages of $\sim 5 \mathrm{Myr}$, clocked by the telltale Wolf-Rayet emission features.

We have subtracted continuum scattered light from the accreting SMBHs before performing stellar population synthesis analysis. We quantify the scattered light by constraining the broad$H \beta$ component underlying the strong narrow $\mathrm{H} \beta$. In luminous obscured quasars, scattered light may contaminate significantly the total observed continuum $(30-60 \%$ at $5100 \AA$; see also Zakamska et al. 2006). While bringing in considerable uncertainties to stellar population analysis, scattered light provides an opportunity to estimate virial black hole masses in obscured quasars.

Measuring stellar velocity dispersions in luminous obscured quasars is challenging even with deep spectroscopy using 8-m class telescopes. In addition to scattered light which dilutes stellar absorption features, there is severe contamination from surrounding emission lines especially around the $\mathrm{Ca} \mathrm{K}$ and $\mathrm{Mg}$ Ib-Fe regions (Greene \& Ho 2006). We obtain stellar velocity dispersion measurements from the $\mathrm{G}$ band, which range from 120 to $420 \mathrm{~km} \mathrm{~s}^{-1}$. These translate into a median black hole mass of $\sim 10^{8.8} M_{\odot}$ assuming the local $M_{\mathrm{BH}}-\sigma_{*}$ relation and a median Eddington ratio of $\sim 0.7$ with a scatter consistent with measurement uncertainties.

The strategy demonstrated is being extended to larger samples and to higher redshifts. It opens up a detailed and unambiguous view to galaxies harboring violently accreting SMBHs, the study of which is crucial to our understanding of the nature and evolution of the coupled growth of galaxies and their central SMBHs.

\section{References}

Greene, J. E. \& Ho, L. C. 2006, ApJ, 641, 117

Liu, X., Zakamska, N. L., Greene, J. E., Strauss, M. A., Krolik, J. H., \& Heckman, T. M. 2009, ApJ, 702, 1098

Zakamska, N. L., et al. 2006, AJ, 132, 1496 